\title{
Analysis of electromagnetic field polarizations in multi-antenna systems
}

Michael A. Jensen

jensen@byu.edu

Jon W. Wallace

wall@ieee.org

Thomas Svantesson

Follow this and additional works at: https://scholarsarchive.byu.edu/facpub

Part of the Electrical and Computer Engineering Commons

\section{Original Publication Citation}

Svantesson, T., M. A. Jensen, and J. W. Wallace. "Analysis of Electromagnetic Field Polarizations in Multiantenna Systems." Wireless Communications, IEEE Transactions on 3.2 (24): 641-6

\section{BYU ScholarsArchive Citation}

Jensen, Michael A.; Wallace, Jon W.; and Svantesson, Thomas, "Analysis of electromagnetic field polarizations in multi-antenna systems" (2004). Faculty Publications. 452.

https://scholarsarchive.byu.edu/facpub/452 


\title{
Analysis of Electromagnetic Field Polarizations in Multiantenna Systems
}

\author{
Thomas Svantesson, Member, IEEE, Michael A. Jensen, Senior Member, IEEE, and Jon W. Wallace, Member, IEEE
}

\begin{abstract}
This paper provides an analytical framework useful for assessing the use of all six electric and magnetic electromagnetic field polarizations for multiantenna communications systems. The approach uses a mapping between the induced signal currents and the received electromagnetic field in order to formulate a diversity interpretation of the six-polarization problem. Application of the framework to a simple, yet representative, channel model demonstrates that for full multipath elevation and azimuthal angle spread, six communication modes are theoretically possible. However, to implement the system requires more antenna design work since a straightforward implementation is found to reduce the potential number of modes to three.
\end{abstract}

Index Terms-Antenna radiation patterns, array antennas, channel capacity, correlation methods, diversity reception, multiple-input multiple-output (MIMO) channels, polarization.

\section{INTRODUCTION}

M ULTIELEMENT antenna systems for wireless communications have demonstrated an ability to provide large capacity gains when used in multipath propagation channels [1]-[3]. Whether used in a diversity scheme or a more advanced multiple-input multiple-output (MIMO) strategy, these systems exploit the various communication modes that exist within the propagation channel. The concept of using electromagnetic wave polarization to provide two communication channels has been understood for some time [4]-[8]. However, recent work has appeared suggesting that each of the six electric and magnetic field vector polarizations can offer an independent channel [9]. These findings have generated considerable interest in the potential of using magnetic field components for communication, but at the same time have raised questions concerning the independence of electric and magnetic fields at a point in space.

In order to provide some insight into the use of all six field polarizations for communication, we introduce an analysis framework for characterizing the correlation behavior of the electromagnetic field components. This analysis is then used to predict the potential performance improvement by employing all

Manuscript received July 3, 2002; revised December 21, 2002; accepted January 28,2003 . The editor coordinating the review of this paper and approving it for publication is R. Murch. This work was supported by the Swedish Foundation for Strategic Research, under the Personal Computing and Communications Program, the Hans Werthén Foundation, and the National Science Foundation under Wireless Initiative Grant CCR-9979452 and Information Technology Research Grant CCR-0081476.

The authors are with the Department of Electrical and Computer Engineering, Brigham Young University, Provo, UT 84602 USA.

Digital Object Identifier 10.1109/TWC.2003.821146 six electromagnetic field polarizations. Although, these predictions are based on theoretical bounds assuming point sensors, they identify fundamental performance limits, an understanding of which is useful when designing actual systems employing multiple polarizations.

The analysis approach utilizes the mapping between induced currents and electromagnetic fields to formulate a joint angle and polarization diversity interpretation of the six received signals. An eigenanalysis of the received signal correlation matrix is used to assess the number of effective communication channels available for different incident field characteristics. The combined performance of these channels is also investigated by calculating the potential channel capacity of a MIMO system using all six possible field sensors.

\section{ANALYSIS FOR IDEAL POINT SENSORS}

To facilitate the study of multimode communications using multiple colocated antennas, we first provide a simple analytical framework for exploring the achievable diversity in realistic channels. We then use the results of this analysis to discuss the communications performance in the context of multiple electric and magnetic field polarizations.

\section{A. Receive Basis Formulation}

In this framework, we will describe the signal received on an antenna system located at a single point in space using induced-vector currents. This approach ensures that we are using a complete "basis" representation, while providing physical insight into the diversity offered by point sensors in a multipath environment. These currents are identified in the point form of Maxwell's equations, which for a (suppressed) $e^{j \omega t}$ time variation are given as [10]

$$
\begin{aligned}
\nabla \times \bar{E} & =-\bar{M}-j \omega \mu \bar{H} \\
\nabla \times \bar{H} & =\bar{J}+j \omega \epsilon \bar{E}
\end{aligned}
$$

where $\bar{E}$ and $\bar{H}$ are electric and magnetic fields, and $\epsilon$ and $\mu$ represent the material permittivity and permeability, respectively. These expressions clearly indicate that, in general, both electric $(\bar{J})$ and magnetic $(\bar{M})$ currents must be specified to uniquely 
TABLE I

ELEMENTS OF THE FIELD PATTERN FOR DIFFERENT RADIATING CURRENTS

\begin{tabular}{l|ccc|ccc}
\hline \hline & \multicolumn{3}{|c|}{ Electric Current } & \multicolumn{3}{|c}{ Magnetic Current } \\
& $\hat{x}$ & $\hat{y}$ & $\hat{z}$ & $\hat{x}$ & $\hat{y}$ & $\hat{z}$ \\
$i$ & 1 & 2 & 3 & 4 & 5 & 6 \\
\hline$E_{i, \theta}$ & $-\cos \theta \cos \phi$ & $-\cos \theta \sin \phi$ & $\sin \theta$ & $\sin \phi$ & $-\cos \phi$ & 0 \\
$E_{i, \phi}$ & $\sin \phi$ & $-\cos \phi$ & 0 & $\cos \theta \cos \phi$ & $\cos \theta \sin \phi$ & $-\sin \theta$ \\
\hline \hline
\end{tabular}

define the fields radiated from a point source. Infinitesimal electric and magnetic ${ }^{1}$ dipoles will be used in the following analysis to represent electric and magnetic current point sources. Practical antennas of finite size will be discussed in Section IV. The complete current basis set consists of the three vector components for each current type. While any coordinate frame can be used to express these vectors, rectangular coordinates will be used in this study.

Using established electromagnetic theory, it is straightforward to transform the six basis currents into a basis consisting of radiated (or received) electric and magnetic fields [10]. In the far field, the radial dependence of $e^{-j k_{o} r} / r$ is identical for all of these fields, and we therefore retain only the angular dependence. The electric far-field radiation patterns for all current elements can be expressed in the form

$$
\bar{E}_{i}(\theta, \phi)=\hat{\theta} E_{i, \theta}+\hat{\phi} E_{i, \phi}
$$

where the components $E_{i, \theta}$ and $E_{i, \phi}$ are shown in Table I. These pattern expressions indicate both the spatial distribution of fields radiated by the current basis and the strength of the current induced when a field is incident on one of the sensors. The $\hat{z}$-oriented current basis in Table I can be found in most antenna textbooks [10]. The remaining patterns can be obtained by either a direct derivation using auxiliary vector potentials or by a coordinate transformation of the expressions for the $\hat{z}$-oriented currents [10]. Furthermore, only the electric far fields are given in Table I since, in the far field, the magnetic field due to each basis current is related to the electric field by a rotation $\bar{H}=(\hat{r} \times \bar{E}) / \sqrt{\mu / \epsilon}$. In the following, we investigate the achievable decorrelation of signals received by each of the current bases despite this dependency between electric and magnetic fields.

\section{B. Channel Description}

Examining the performance of systems exploiting multiple electromagnetic polarizations requires formulation of a channel model that is adequately descriptive yet simple. We, therefore, consider the electromagnetic field at a point in space to be a combination of plane waves according to the relation

$$
\bar{E}=\int \bar{e}(\Omega) d \Omega
$$

where $\bar{e}$ gives the amplitude, phase, and polarization of a plane wave propagating in the solid angle direction $\Omega$ (azimuth and elevation). We will represent this incident field by its statistical properties over an ensemble of propagation scenarios. For simplicity of analysis and illustration of the key mechanisms cre-

\footnotetext{
${ }^{1}$ While magnetic currents can not be generated physically, it is well known that the fields from such a current can be constructed using an infinitesimal current loop [10].
}

ating diversity, we will assume that for each plane-wave component the two orthogonal polarizations are uncorrelated and that plane-wave components separated in angle are also uncorrelated. These assumptions are represented symbolically as [11]

$$
\begin{aligned}
\mathcal{E}\left\{e_{\theta}(\Omega) e_{\phi}^{*}\left(\Omega^{\prime}\right)\right\} & =0 \\
\mathcal{E}\left\{e_{\phi}(\Omega) e_{\theta}^{*}\left(\Omega^{\prime}\right)\right\} & =0 \\
\mathcal{E}\left\{e_{\theta}(\Omega) e_{\theta}^{*}\left(\Omega^{\prime}\right)\right\} & =S_{\theta}(\Omega) \delta\left(\Omega-\Omega^{\prime}\right) \\
\mathcal{E}\left\{e_{\phi}(\Omega) e_{\phi}^{*}\left(\Omega^{\prime}\right)\right\} & =S_{\phi}(\Omega) \delta\left(\Omega-\Omega^{\prime}\right)
\end{aligned}
$$

where $\mathcal{E}\{\cdot\}$ indicates an expectation and $S_{\theta}$ and $S_{\phi}$ represent the average angular power densities (Watts/steradian) of the $\hat{\theta}$ and $\hat{\phi}$ polarizations of the incident field. In the following, we will assume that $S_{\theta}(\Omega)=S_{\phi}(\Omega)=S(\Omega)$.

\section{Receive Diversity Performance}

The radiation patterns of (2) can be used in conjunction with established correlation analysis to determine the achievable diversity performance of a point receiver. To this end, consider that the signal on the $i$ th receive element is given as

$$
y_{i}=\int_{\Delta \Omega} \bar{e}(\Omega) \cdot \bar{E}_{i}(\Omega) d \Omega
$$

where $\Delta \Omega$ represents the angular range of arrival angles for the incident field. The key point indicated in (5) is that the incoming field is weighted differently by each receiving radiation pattern according to the field's angular and polarization properties. Stated another way, the received signal is simply a projection of the incident field upon the receiving radiation pattern. If the receiving patterns are orthogonal, the received signals will be uncorrelated [11]. Using the conditions in (4), the correlation between the signals from the $i$ th and $k$ th receive elements becomes [11], [12]

$$
R_{i k}=\mathcal{E}\left\{y_{i} y_{k}^{*}\right\}=\int_{\Delta \Omega} S(\Omega) \bar{E}_{i}(\Omega) \cdot \bar{E}_{k}^{*}(\Omega) d \Omega .
$$

For the computations that follow, we make a final assumption that the incident power density $S(\Omega)$ is constant over an angular region defined by $\Delta \Omega$ and zero otherwise. This allows assessment of the impact of angular spread on the received signal correlation while being consistent with measurements and published channel models. Thus, both the azimuth and elevation angles are uniformly distributed within an angular region characterized by $|\theta-\pi / 2| \leq \Delta \theta / 2$ in elevation and $|\phi| \leq \Delta \phi / 2$ in azimuth. For this case, the values of $R_{i k}$ can be computed analytically and the correlation matrix $\mathbf{R}$ becomes

$$
\mathbf{R}=\left[\begin{array}{cccccc}
R_{11} & 0 & 0 & 0 & 0 & 0 \\
0 & R_{22} & 0 & 0 & 0 & R_{26} \\
0 & 0 & R_{33} & 0 & -R_{26} & 0 \\
0 & 0 & 0 & R_{11} & 0 & 0 \\
0 & 0 & -R_{26} & 0 & R_{22} & 0 \\
0 & R_{26} & 0 & 0 & 0 & R_{33}
\end{array}\right]
$$

where

$$
\begin{aligned}
R_{11}= & \frac{3}{4 \pi} \Delta \phi \sin \left(\frac{\Delta \theta}{2}\right)-\frac{3}{32 \pi}[\Delta \phi+\sin (\Delta \phi)] \\
& \times\left[3 \sin \left(\frac{\Delta \theta}{2}\right)+\frac{1}{3} \sin \left(\frac{3 \Delta \theta}{2}\right)\right]
\end{aligned}
$$




$$
\begin{aligned}
R_{22}= & \frac{3}{4 \pi} \Delta \phi \sin \left(\frac{\Delta \theta}{2}\right)-\frac{3}{32 \pi}[\Delta \phi-\sin (\Delta \phi)] \\
& \times\left[3 \sin \left(\frac{\Delta \theta}{2}\right)+\frac{1}{3} \sin \left(\frac{3 \Delta \theta}{2}\right)\right] \\
R_{33}= & \frac{3}{16 \pi} \Delta \phi\left[3 \sin \left(\frac{\Delta \theta}{2}\right)+\frac{1}{3} \sin \left(\frac{3 \Delta \theta}{2}\right)\right] \\
R_{26}= & \frac{3 \sin \left(\frac{\Delta \phi}{2}\right)}{8 \pi}[\Delta \theta+\sin (\Delta \theta)] .
\end{aligned}
$$

Most entries in the correlation matrix in (7) are zero due to orthogonality of the radiation patterns. Note that when $\Delta \theta=0$, we must instead represent the incident power density as $S(\Omega)=S(\phi) \delta(\theta-\pi / 2)$. This leads to simplified expressions for (8)-(11).

Diversity performance can be quantified in terms of the number and quality of independent spatial communication modes made available by the channel and receive antenna configuration. The eigenvalues $\lambda_{i}, 1 \leq i \leq 6$, of the correlation matrix $\mathbf{R}$, provide a measure of this performance and will therefore be examined for this study. For the correlation matrix in (7), the eigenvalues can be calculated in closed form as

$$
\begin{aligned}
& \lambda_{1,2}=R_{11} \\
& \lambda_{3,4}=\frac{1}{2}\left[R_{22}+R_{33}+R_{\delta}\right] \\
& \lambda_{5,6}=\frac{1}{2}\left[R_{22}+R_{33}-R_{\delta}\right]
\end{aligned}
$$

where $R_{\delta}=\sqrt{R_{22}^{2}-2 R_{22} R_{33}+R_{33}^{2}+4 R_{26}^{2}}$. It is interesting to note that each eigenvalue has a multiplicity of two, leading to at most three distinct eigenvalues. A physical interpretation of this can be found in the radiation patterns in Table I. The patterns corresponding to magnetic currents $(i=4$ to 6 ) are functionally identical to-yet vectorially orthogonal to - the patterns corresponding to electric currents $(i=1$ to 3 ). Thus, the different patterns give rise to three distinct eigenvalues, and the vector orthogonality creates the multiplicity under the assumption that the incident field has equal power in the two orthogonal polarizations.

Optimal diversity performance will result when $\mathbf{R}$ has six equal (nonzero) eigenvalues. Typically, this occurs for a covariance matrix $\mathbf{R}=\alpha \mathbf{I}$, where $\mathbf{I}$ is the identity matrix and $\alpha$ is a constant. This form indicates zero cross correlation and perfect power balance across the sensor. In order to remove the effect of the total received power for a given computation, we will plot the normalized eigenvalues

$$
\hat{\lambda}_{i}=\frac{\lambda_{i}}{\sum_{j} \lambda_{j}} .
$$

Fig. 1 shows the variation of the normalized eigenvalues with angular spread parameter $\Delta \phi$ for two different values of $\Delta \theta$. Note that each curve actually represents two eigenvalues, as mentioned above. As can be seen, for a single propagation path ( $\Delta \theta=\Delta \phi=0$ ), only two channels exist corresponding to the two possible polarizations of the incident plane wave. As the angle spread increases, the remaining four eigenvalues increase. However, for zero elevation spread, the eigenvalues do not reach the same value. For the case of full elevation spread $\Delta \theta=180^{\circ}$,
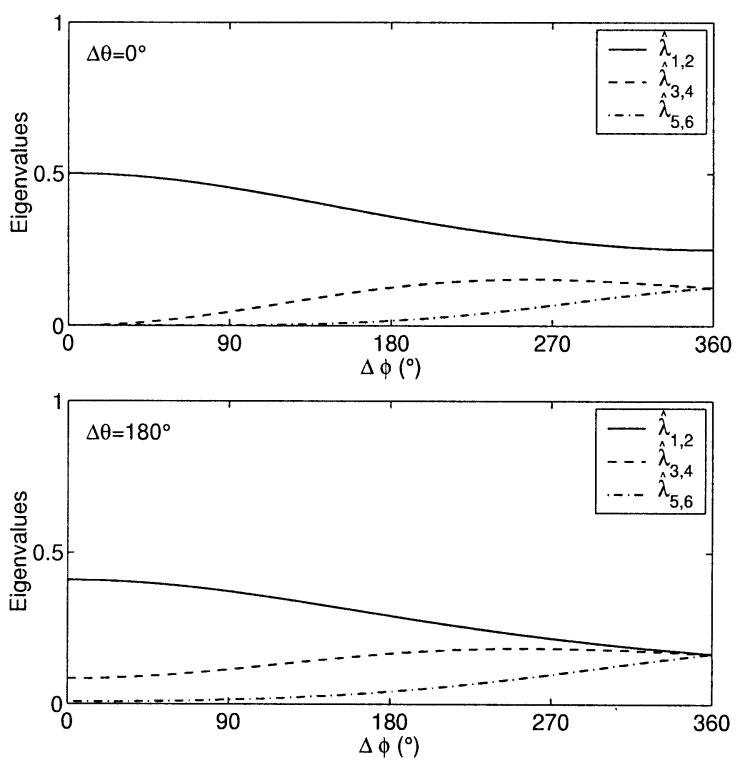

Fig. 1. Normalized eigenvalues of the correlation matrix versus incident field angle spread parameters assuming ideal point sensors. Each curve represents two degenerate eigenvalues.

all eigenvalues tend toward the same value, indicating the availability of six independent communication modes. Because observed elevation spread in indoor and urban environments is relatively small [13], the curve for $\Delta \theta=0$ is particularly interesting. Most noteworthy for this case is the fact that if full azimuthal spread is considered, the correlation matrix becomes diagonal indicating six independent channels. These channels, however, are not all equally "good" since the power received by the $\hat{z}$ oriented sensors is twice as large as the power received by the other sensors [14].

The eigenvalues of the correlation matrix $\mathbf{R}$ can be used to quantify the diversity performance of this point sensor configuration. Specifically, in [15], an effective number of diversity branches was calculated from the eigenvalues. To understand this measure, let the instantaneous signal-to-noise ratio (SNR) for maximal ratio (MR) combined signals from the antennas be represented as $\gamma(p \%)$, where $p \%$ denotes a given probability level on the cumulative probability distribution function (CDF). The diversity gain for $N$ branches is then defined as

$$
\mathrm{DG}^{(p \%)}(N)=\frac{\left.\gamma(p \%)\right|_{n=N}}{\left.\gamma(p \%)\right|_{n=1}} .
$$

Assuming that all branches are uncorrelated and have equal average SNR $\Gamma=\mathcal{E}\{\gamma\}$, then this diversity gain can be computed using the CDF of the MR combined signals given in [14] as

$$
p_{\mathrm{MR}}(\gamma \leq x)=1-e^{-x / \Gamma} \sum_{k=1}^{N} \frac{\left(\frac{x}{\Gamma}\right)^{k-1}}{(k-1) !} .
$$

When assessing the diversity gain of correlated antenna branches, we create an effective set of uncorrelated branches, where the generally unequal average SNRs of the branches are given by the eigenvalues of $\mathbf{R}$. The cumulative probability function for this scenario was derived in [15] for the case of distinct eigenvalues. In this study, each eigenvalue in (12) was found to have a multiplicity of two. However, by using the same 


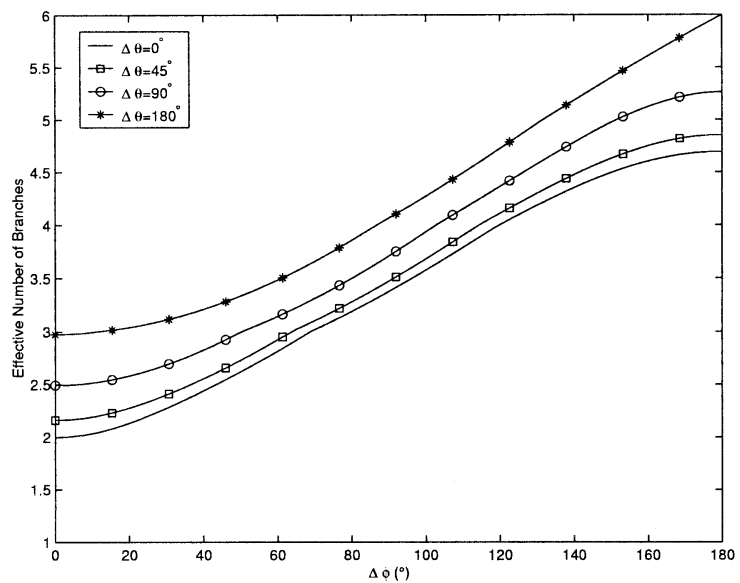

Fig. 2. Effective number of diversity branches assuming ideal point sensors for various values of $(\Delta \theta, \Delta \phi)$.

derivation approach as in [15], the CDF for the MR combined instantaneous SNR $\gamma$ for $N$ uncorrelated branches with unequal SNRs (but with multiplicity two) can be found to be

$$
\begin{aligned}
p_{\mathrm{MR}}(\gamma \leq x)=1 & +\sum_{l=1}^{N / 2} \frac{e^{-x / \Gamma_{l}}}{\prod_{k=1}^{N / 2} \Gamma_{k}^{2} \prod_{m \neq l}\left(\frac{-1}{\Gamma_{l}}+\frac{1}{\Gamma_{m}}\right)^{2}} \\
& \times\left[-x \Gamma_{l}-\Gamma_{l}^{2}+2 \Gamma_{l} \sum_{m \neq l} \frac{1}{\frac{-1}{\Gamma_{l}}+\frac{1}{\Gamma_{m}}}\right]
\end{aligned}
$$

where $m=(1,2, \ldots, N / 2)$ and $\Gamma_{1}, \Gamma_{2}, \ldots, \Gamma_{N / 2}$ denote the branch SNRs. The diversity gain for the six basis currents $(N=6), \mathrm{DG}^{(p \%)}(6)$ is now calculated using (18) and (16). To compute the effective number of diversity branches, we find the number $N_{B}$ of uncorrelated branches with equal average SNR required to obtain $\mathrm{DG}_{R}^{(p \%)}\left(N_{B}\right)=\mathrm{DG}^{(p \%)}(6)$, where $\mathrm{DG}_{R}^{(p \%)}\left(N_{B}\right)$ is the reference diversity gain computed using (17) and (16). To obtain fractional values of $N_{B}$, interpolation of the diversity gain as a function of the number of branches was performed.

Fig. 2 shows the number of effective branches assuming point sensors for various angular spreads. As expected, for $(\Delta \theta=$ $\Delta \phi=0$ ) there are only two branches due to polarization orthogonality. However, by increasing the azimuth spread, the number of effective branches increases due to the decreasing correlation created by angle diversity [see (8)-(11)]. For full angular spread, the maximum of six branches is achieved as found in the eigenvalue analysis. It is also interesting to note that even with a small elevation spread, more than four effective branches are obtained for large azimuth spreads.

\section{Channel Capacity}

The different electromagnetic field polarizations can also be used to obtain a compact MIMO system capable of achieving high data rates. To investigate the potential of using all six vector current sensors, the antenna arrangement using three infinitesimal electric and three magnetic dipoles will be used at both the transmitter and receiver. The orthogonally oriented dipoles are aligned with the Cartesian $x, y$, and $z$. An upper bound on the achievable data rate, the channel capacity, will be used as a performance measure. The instantaneous channel capacity of a narrowband MIMO channel can be written as [2]

$$
C=\log _{2} \operatorname{det}\left[\mathbf{I}+\frac{\rho}{N_{T}} \mathbf{H} \mathbf{H}^{H}\right]
$$

where $\rho$ denotes the SNR at each receive antenna. The element $\mathbf{H}_{i j}$ represents the complex path gain from transmitter $j$ to receiver $i$. Note that the channel matrix $\mathbf{H}$ is $N_{R} \times N_{T}$, where $N_{R}, N_{T}$ denotes the number of antenna elements at the receiver and transmitter, respectively. In our case, $N_{R}=N_{T}=6$. A simple channel model assuming Gaussian distributed channel coefficients will be used to simulate the system. It is further assumed that the correlation properties at transmit and receive are separable. In this case, the overall correlation between the path gain $\mathbf{H}_{i j}$ and $\mathbf{H}_{m n}$ is found as the product of the correlation on the transmit side between element $j$ and $n$ and the correlation on the receive side between element $i$ and $m$. This has been found to be a reasonable assumption for well-separated transmit and receive arrays [16], [17]. Using these assumptions, the total correlation matrix can be written as the Kronecker product between the transmit and receive correlation matrices or $\mathbf{R}_{\text {tot }}=\mathbf{R}_{R} \otimes \mathbf{R}_{T}$ [16], [17], and the distribution of the stacked channel coefficients becomes vec $(\mathbf{H}) \in \mathrm{N}\left(0, \mathbf{R}_{\text {tot }}\right)$. The elements of the transmit correlation matrix are defined as $\mathbf{R}_{T i j}=\mathcal{E}\left\{\mathbf{H}_{\cdot i} \mathbf{H}_{\cdot j}\right\}$ and the elements of the receive correlation matrix are defined as $\mathbf{H}_{R i j}=\mathcal{E}\left\{\mathbf{H}_{i} \cdot \mathbf{H}_{j}\right\}$. The channel is also normalized such that $\|\mathbf{H}\|_{F}^{2}=\operatorname{Tr}\left(\mathbf{H}^{H} \mathbf{H}\right)=\sqrt{N_{R} N_{T}}$, where $\operatorname{Tr}(\cdot)$ denotes the trace operator.

Using the above model and assumptions, the average channel capacity is evaluated via the Monte Carlo method. The correlation matrices obtained in Section III were used in these simulations for $\mathbf{R}_{T}$ and $\mathbf{R}_{R}$. In Fig. 3, the average channel capacity of $10^{4}$ channel realizations is shown for various values of $(\Delta \theta, \Delta \phi)$. The same spreads were used at both the transmitter and receiver side. As expected, the capacity grows as the angular spread increases. Relatively large vertical spreads are needed to lift the capacity when the azimuth spread is low. Furthermore, it is interesting to note that an increase in elevation spread does not yield a significant capacity gain for large azimuth spreads. This implies that the power imbalance observed in the correlation analysis does not severely affect the capacity. This is encouraging since measurement campaigns have revealed relatively small elevation spreads but large azimuth spreads in indoor and pico-cell environments [13]. Such environments are particularly relevant for MIMO systems since they are likely locations for wireless high-speed data links.

\section{DIVERSITY INTERPRETATION}

It is interesting to examine the preceding results within a $d i$ versity context. Consider an incident field consisting of discrete plane waves. The electric and magnetic fields at the observation point may be expressed as

$$
\begin{aligned}
& \bar{E}\left(\bar{r}_{o}\right)=\sum_{m}\left(A_{m} \hat{\theta}+B_{m} \hat{\phi}\right) e^{-j k_{o} \hat{r}_{m} \cdot \bar{r}_{0}} \\
& \bar{H}\left(\bar{r}_{o}\right)=\frac{1}{\eta_{o}} \sum_{m}\left(-B_{m} \hat{\theta}+A_{m} \hat{\phi}\right) e^{-j k_{o} \hat{r}_{m} \cdot \bar{r}_{0}}
\end{aligned}
$$




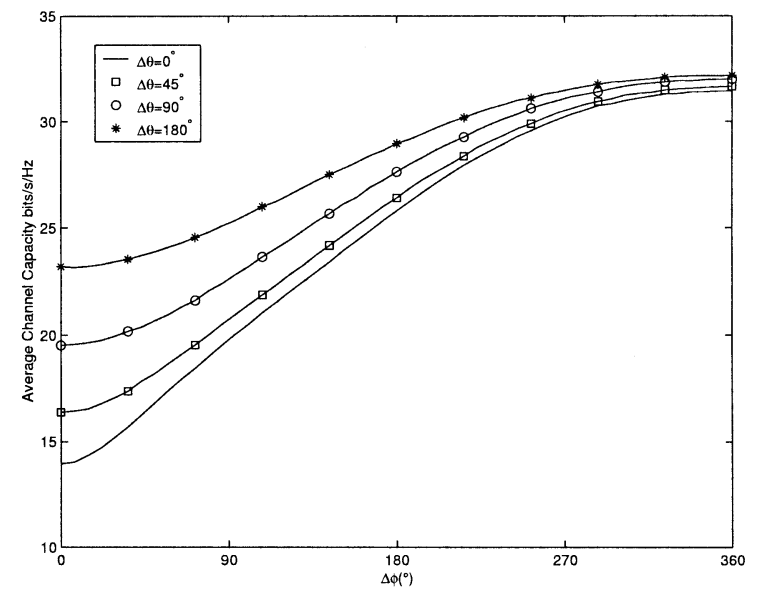

Fig. 3. Average channel capacity assuming ideal point sensors for various values of $(\Delta \theta, \Delta \phi)$ and $\rho=20 \mathrm{~dB}$.

where $k_{o}$ and $\eta_{o}$ are the free-space wavenumber and intrinsic impedance, respectively, and $\hat{r}_{m}$ represents the unit vector in the arrival direction for the $m$ th incident plane wave. These expressions indicate that the magnetic fields do not provide any information not contained within the electric field. In particular, if there is only one wave $(m=1)$, the two polarizations create the two communication modes, and the resulting diversity is therefore referred to as polarization diversity. Note that there are only two nonzero eigenvalues and two effective diversity branches in this special case, as found in Figs. 1 and 2.

Despite the observation that the incident magnetic field does not provide any new information, we still found in Section II-C that the signals received by the electric sensors can be uncorrelated with the signals received by the magnetic sensors. In fact, if there is rich multipath, all six signals computed according to (5) were found to be uncorrelated and of equal strength. The key in obtaining the decorrelation is to observe the projection of the incident field onto each basis function in (5). The result of this operation is that each sensor applies a unique magnitude weighting (the radiation pattern) to the multipath components based upon their arrival angles, and the resulting diversity can therefore be appropriately referred to as pattern diversity [11]. Thus, it is not the incoming electric and magnetic field that are uncorrelated; instead, it is the fact that each received signal is weighted by an orthogonal pattern that provides the diversity. Hence, the total diversity order of six is achieved by a combination of angle and polarization diversity. Finally, it should be noted that the weighting using different radiation patterns is analogous to the operation performed by spatially separated antennas which uniquely weight the phases of the multipath arrivals.

\section{CONCLUSION AND Discussion}

This paper has presented an analytical framework useful for assessing the behavior of signals received by orthogonally oriented field sensors at a point in space. The formulation uses field basis functions derived from vector current basis functions in order to determine the receive signal correlation matrix for a given incident multipath field. This interpretation suggests that a combination of angle and polarization diversity, enabled by the six possible current components, can lead to up to six degrees of communication freedom for ideal point sensors. The channel capacity for systems employing multiple electromagnetic field polarizations was also investigated. It was found that a large portion of the potential capacity can be obtained with limited elevation spread as long as the azimuth spread is relatively large which are common conditions in indoor and urban environments. It is hoped that this analysis will provide insight into the physical behavior leading to multiple communication modes based upon electromagnetic field polarization.

The analysis of the potential performance of employing multiple electromagnetic polarizations was based on ideal point sensors. From a practical standpoint, however, antennas must be of finite size. To examine the performance of real antennas, a straightforward antenna solution was implemented using half-wave dipoles and full-wave loops to approximate the ideal point sensors. These antenna topologies were chosen since they are easily matched to the transmit/receive system over reasonable communication bandwidths. However, the radiation patterns of this antenna arrangement were found to be nonorthogonal. In fact, in a rich multipath environment only three significant eigenvalues was found. This arrangement has about half the effective number of diversity branches and slightly more than half the channel capacity of the ideal antenna arrangement analyzed in this paper. Thus, finding efficient compact antennas that exhibit radiation patterns that give low correlation and high capacity is an interesting and important antenna design problem that recently has received more attention [18].

\section{REFERENCES}

[1] J. H. Winters, "On the capacity of radio communication systems with diversity in a rayleigh fading environment," IEEE J. Select. Areas Commun., vol. SAC-5, pp. 871-878, June 1987.

[2] G. J. Foschini and M. J. Gans, "On limits of wireless communications in a fading environment when using multiple antennas," Wireless Personal Commun., vol. 6, pp. 311-335, Mar. 1998.

[3] G. G. Raleigh and J. M. Cioffi, "Spatio-temporal coding for wireless communication," IEEE Trans. Commun., vol. 46, pp. 357-366, Mar. 1998.

[4] R. G. Vaughan, "Polarization diversity in mobile communications," IEEE Trans. Veh. Technol., vol. 39, pp. 177-186, Aug. 1990.

[5] D. C. Cox, "Antenna diversity performance in mitigating the effects of portable radiotelephone orientation and multipath propagation," IEEE Trans. Commun., vol. 31, pp. 620-628, May 1983.

[6] B. Friedlander and A. J. Weiss, "Performance of diversely polarized antenna arrays for correlated signals," IEEE Trans. Aerospace Electron. Syst., vol. 28, pp. 869-879, July 1992.

[7] J. P. Kermoal, L. Schumacher, F. Frederiksen, and P. E. Mogensen, "Polarization diversity in MIMO channels: Experimental validation of a stochastic model and performance assessment," in IEEE VTC'2001 Fall Conf., vol. 2, Atlantic City, NJ, Oct. 7-11, 2001, pp. 1078-1082.

[8] L. C. Lukama, K. Konstantinou, and D. J. Edwards, "Performance of a three-branch orthogonal polarization diversity scheme," in IEEE VTC'2001 Fall Conf., vol. 2, Atlantic City, NJ, Oct. 7-11, 2001, pp. 1078-1082.

[9] R. A. Andrews, P. P. Mitra, and R. deCarvalho, "Tripling the capacity of wireless communications using electromagnetic polarization," Nature, vol. 409, pp. 316-318, Jan. 2001.

[10] C. A. Balanis, Antenna Theory: Analysis and Design. New York: Wiley, 1997.

[11] R. G. Vaughan and J. B. Andersen, "Antenna diversity in mobile communications," IEEE Trans. Veh. Technol., vol. 36, pp. 149-172, Nov. 1987.

[12] M. A. Jensen and Y. Rahmat-Samii, "Performance analysis of antennas for hand-held transceivers using FDTD," IEEE Trans. Antennas Propagat., vol. 42, pp. 1106-1113, Aug. 1994. 
[13] L. M. Correia, Wireless Flexible Personalised Communications: Wiley, 2001.

[14] W. C. Jakes, Microwave Mobile Communications: IEEE Press, 1993.

[15] O. Nørklit, P. D. Teal, and R. G. Vaughan, "Measurement and evaluation of multi-antenna handsets in indoor mobile communication," IEEE Trans. Antennas Propagat., vol. 49, pp. 429-437, Mar. 2001.

[16] J. P. Kermoal, L. Schumacher, K. I. Pedersen, P. E. Mogensen, and F. Fredriksen, "A stochastic mimo radio channel model with experimental validation," IEEE J. Select. Areas Commun., vol. 20, pp. 1211-1226, Aug. 2002.

[17] K. Yu, M. Bengtsson, B. Ottersten, D. McNamara, P. Karlsson, and M. Beach, "Second order statistics of nlos indoor mimo channels based on 5.2ghz measurements," Proc. IEEE Globecom 2001, vol. 1, pp. 156-160, Nov. 2001.

[18] S. C. K. Ko and R. D. Murch, "Compact integrated diversity antenna for wireless communications," IEEE Trans. Antennas Propagat., vol. 49, pp. 954-960, June 2001.

Thomas Svantesson (S'98-M'01) was born in Trollhättan, Sweden, in 1972. He received the M.S. degree in electrical engineering in 1996 and the Ph.D. degree in signal processing in 2001 from Chalmers University of Technology, Göteborg, Sweden.

From 2001 to 2002, he was a Postdoctoral Researcher at the Brigham Young University, Provo, UT. Presently, he is with the same department conducting research on adaptive antennas in wireless communications, channel modeling, and probing of systems employing transmit and receive antenna arrays.
Michael A. Jensen (S'93-M'95-SM'01) received the B.S. (summa cum laude) and M.S. degrees in electrical engineering from Brigham Young University (BYU), Provo, UT, in 1990 and 1991, respectively, and the Ph.D. in electrical engineering at the University of California, Los Angeles (UCLA), in 1994.

From 1989 to 1991, he was a Graduate Research Assistant in the Lasers and Optics Laboratory at BYU. In 1990, he received a National Science Foundation Graduate Fellowship. From 1991 to 1994, he was a Graduate Student Researcher in the Antenna Laboratory at UCLA. Since 1994, he has been at the Electrical and Computer Engineering Department, BYU, where he is currently an Associate Professor. His research interests include antennas and propagation for personal communications, microwave circuit design, radar remote sensing, numerical electromagnetics, and optical fiber communications. He is a member of Eta Kappa Nu and Tau Beta Pi.

Dr. Jensen currently serves on the Joint Meetings Committee for the IEEE Antennas and Propagation Society and served as the Technical Program Chair for the 2000 IEEE International Symposium on Antennas and Propagation. He was awarded the best paper award at the 1994 IEEE International Symposium on Antennas and Propagation.

Jon W. Wallace (S'01-M'03) received the B.S. (summa cum laude) and Ph.D. degrees in electrical engineering from Brigham Young University, Provo, UT, in 1997 and 2002, respectively.

From 1995 to 1997, he worked as an Associate of Novell, Inc., Provo. During 1997, he was a Member of Technical Staff for Lucent Technologies in Denver, CO. He received the National Science Foundation Graduate Fellowship in 1998. From 1998 to 2002, he worked as a Graduate Student Researcher in the BYU Wireless Communications Laboratory. He is currently a Research Associate of the BYU Wireless Communications Laboratory and works in Vienna, Austria, pursuing joint research with the Technical University of Vienna Mobile Communications Group. His research interests include wireless channel sounding and modeling, optical device modeling, and remote sensing. 